\title{
Analog-Circuit-Based Activities to Improve Introductory Continuous-Time Signals and Systems Courses
}

Dr. Mario Simoni, Rose-Hulman Institute of Technology

Dr. Simoni is an Associate Professor of Electrical and Computer Engineering at Rose-Hulman Institute of Technology in Terre Haute, IN.

Prof. Maurice F. Aburdene, Bucknell University

Maurice Felix Aburdene is a Professor of Electrical Engineering and Professor of Computer Science at Bucknell University. His teaching and research interests include control systems, parallel algorithms, simulation of dynamic systems, and signal processing.

Ms. Farrah Fayyaz, Purdue University

Graduate Student 


\title{
Analog-Circuit-Based Activities to Improve Introductory Continuous-Time Signals and Systems Courses
}

\begin{abstract}
The introductory continuous-time signals and systems course (CTSS) is one of the most difficult courses that students encounter in an electrical and computer engineering (ECE) curriculum, as evidenced by well-above-average drop/failure rates. This paper presents a series of analog-circuit based activities that can help students visualize the complex mathematical concepts and gain a better appreciation for how the concepts are useful in real-world situations. These exercises have been used in an introductory CTSS course since September of 2010. While the exercises were designed to be done in a three-hour session, we show how they can be broken up into smaller steps that could be completed easily in a one-hour class either by the students or as a demonstration by the professor. In order to get some measure of the impact of these exercises, the students were surveyed for one year before and also since the exercises were introduced. The results from this survey and also the Continuous-Time Signals and Systems Concept Inventory are presented as a measure of the impact of these exercises on the students' affective and cognitive learning experience.
\end{abstract}

\section{Introduction}

The introductory continuous-time signals and systems (CTSS) course is one of the most difficult courses that students encounter in an electrical and computer engineering (ECE) curriculum, as evidenced by well-above-average drop/failure rates ${ }^{1-4}$. The foundation of Fourier theory with regard to signal analysis is the understanding of how sinusoids compose periodic signals. The highly mathematical, abstract, and theoretical concepts are difficult for the students to visualize. Most students have no conscious personal experience with this phenomenon, so when the theory is described, they have no foundation on which to build.

We are trying to improve motivation and learning by creating application-oriented hands-on active-learning opportunities for students ${ }^{3,4}$. There are many examples of such opportunities described in the literature, but most of these activities make use of MATLAB ${ }^{\circledR}$, LabVIEW $^{\circledR}$, or DSP hardware ${ }^{5}$. The exercises described in this paper are based on analog circuits and their applications. There are certainly advantages to using software simulation tools for laboratory exercises because they are relatively inexpensive compared to laboratory equipment, and once learned, students can very quickly perform a large number of "experiments". In fact, many of the exercises described in this paper could be simulated entirely using software tools. We are not advocating the elimination of these software simulators, and in many cases use them for both prelab exercises and/or analysis of results. However, there is one key advantage to using hardware-based hands-on activities with real-world applications that simply cannot be obtained with software-only based activities. Practicing engineers use the theoretical and mathematical concepts from CTSS courses to model real-world physical phenomena. The overwhelming majority of students have never had an opportunity to connect those theoretical concepts with the physical phenomena that they are trying to model. So they have trouble understanding why they 
are learning the concepts or what use those concepts have. While software tools can help students visualize the concepts, they are in-themselves also modeling tools. An important way for students to gain an experience of the phenomena that they are trying to model is to actually experiment with those physical systems and real-world applications. Using a real system with real-world applications creates a degree of credibility and relevance that is not possible with software simulations ${ }^{6,7}$. Aspects such as nonlinearity and bandwidth limitations are natural parts of the system and, therefore, don't have to be artificially added to a simulation. Analog systems are also truly continuous-time. While sampling artifacts may impact the measurements due to modern instrumentation, they do not impact the actual system. When adjusting parameters in an experiment, the students are not just changing a number, they can tie that parameter to a physical aspect of the system and observe the results in real-time in both the time and frequency domains simultaneously.

While there are many advantages to using analog circuits, building and debugging a circuit can be quite a challenge. In order to facilitate these exercises, an analog-circuit platform was developed at Rose-Hulman Institute of Technology and has been used in the introductory CTSS course, ECE300, since the Fall Quarter of 2010-2011. The signals and systems exploration platform (SSEP) is a printed circuit board (PCB) that can easily be configured to sum, multiply, filter, and sample continuous-time signals. With an onboard microphone, ECG/instrumentation amplifier, and generic signal input, a wide variety of signals can be studied and manipulated. The SSEP platform was described previously, which is why this paper focuses on the laboratory exercises $^{3}$. Figure 1 shows the SSEP and students using it to measure and filter their own ECG in one of the laboratory exercises.
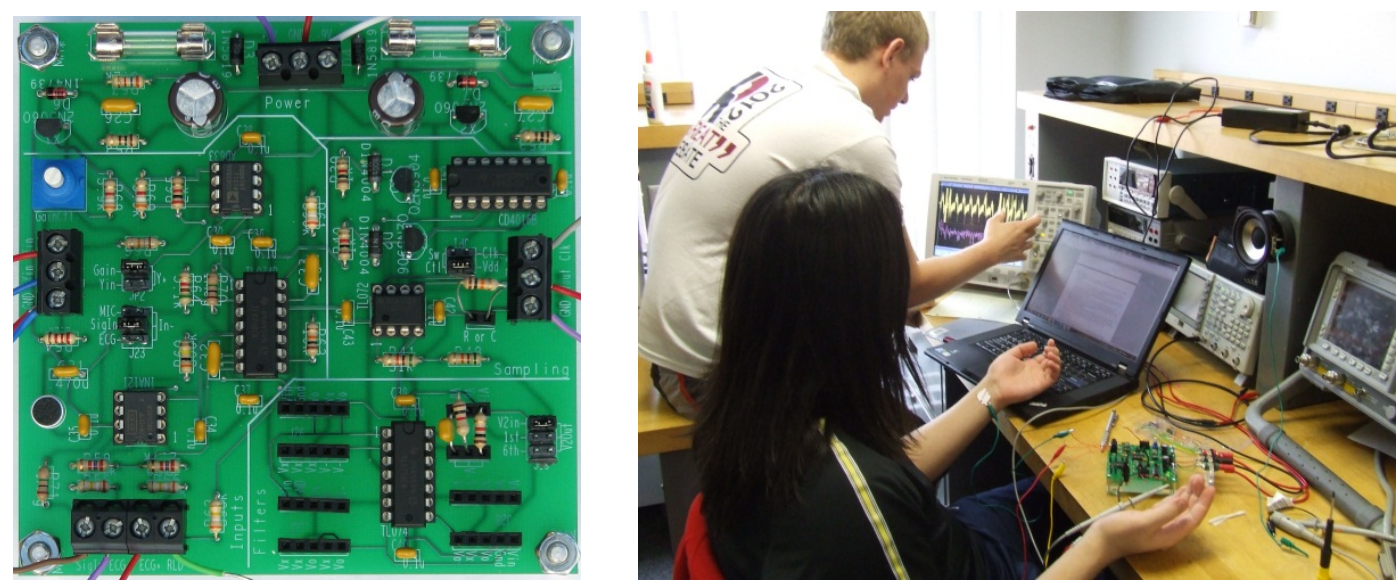

Figure 1: The photo on the left is a top-down view of the SSEP. The photo on the right shows two students measuring their own ECG using the SSEP, which can be seen between the seated-student's hands. The other student is adjusting the oscilloscope, which is recording the ECG from the output terminal of the SSEP. This experiment is described in the Subsection Lab 3: Filtering Periodic Signals.

This paper describes the series of laboratory exercises that were developed for use with the SSEP. While the SSEP platform facilitates the exercises, they can also be performed in traditional labs with commonly available equipment. The important point about the exercises is not how they are done, but that they force the students to connect the theoretical concepts to realworld applications using hands-on activities with physical systems. ECE300 is taught in a 10 week quarter with 3 one-hour lectures and 1 three-hour laboratory session per week. While the 
exercises were designed to be done in a three-hour session, some examples are provided to show how they could be completed easily in a one-hour class either by the students or as a demonstration by the professor. Each of the laboratory sessions follows a similar format by starting with simple examples, such as single sinusoids, and then progressing to real-world applications, such as dual-tone multi-frequency (DTMF) signals and filtering the student's own ECG signal.

These laboratory exercises have been used at Rose-Hulman since the Fall Quarter of the 20102011 academic year. Prior to implementing these exercises, the laboratory portion of the course was based primarily upon MATLAB ${ }^{\circledR}$ programming assignments. In order to get some measure of the impact of these exercises on student perceptions of the course, the students were surveyed for one year before the exercises were introduced. In order to assess the impact on student learning, the normalized gain from the Continuous-Time Signals and Systems Concept Inventory exam will be analyzed from before and after the laboratory exercises were introduced.

\section{The laboratory exercises}

A summary of the exercises that is organized by week is shown in Table I. A general description of the activities for each lab is presented in this section. Detailed descriptions with specific signal parameters, instructions, and questions posed to the students can be obtained by contacting the authors. Because Rose-Hulman is on a quarter system, the breadth of material in ECE300 is more limited than what would be seen in a similar semester course at another institution. ECE300 focuses primarily on the frequency domain analysis of signals and systems and then applications of the frequency domain, such as filters and sampling. Time domain analysis of signals and systems, such as convolution, linearity, and time-invariance, is discussed in ECE205, which is a pre-requisite course to ECE300. One of the required follow-on courses to ECE300 is a discrete-time signal processing course, which is why ECE300 ends with sampling theory. Other pre-requisite courses include the math sequence through differential equations and DC/AC circuits courses. While most students have had an introductory electronics course before taking ECE300, a background in analog electronics is not assumed nor required to perform these exercises.

These exercises are performed in a dedicated laboratory room with standard bench-top laboratory equipment. There are 15 benches in the room, each of which has a power supply, a true RMS DMM, either two function generators or one dual output function generator, an oscilloscope, and a spectrum analyzer. While the exercises were designed with this equipment in mind, they have also been attempted successfully with less sophisticated equipment such as the National Instruments MyDAQ ${ }^{\circledR}$ and the Digilent Electronics Explorer Board ${ }^{\circledR}$. Because most analog circuits have a high input impedance, a laptop's sound card output can be used in combination with MATLAB ${ }^{\circledR}$ to create a makeshift function generator.

The students typically work on the laboratory exercises in pairs and the class sizes have ranged from 16 to 32 students. Each laboratory exercise is handled in a similar manner. At the beginning of the week, the students are given a handout that describes: 1) the general theory for which the lab is intended to illustrate, 2) a pre-lab exercise that usually involves MATLAB ${ }^{\circledR}$ modeling and from which the students must get values that are verified in the lab session, 3) step-by-step instructions for completing the exercises, and 4) data tables and questions that must be 
completed by the students in a post-lab memo. The students are asked to return a memo by the following session. The memo should include screen captures from the oscilloscope and spectrum analyzer, descriptions of the measurements and screen captures, and short answers to any questions that were posed in the handout.

TABLE I

List of labs including the types of signals and systems being used

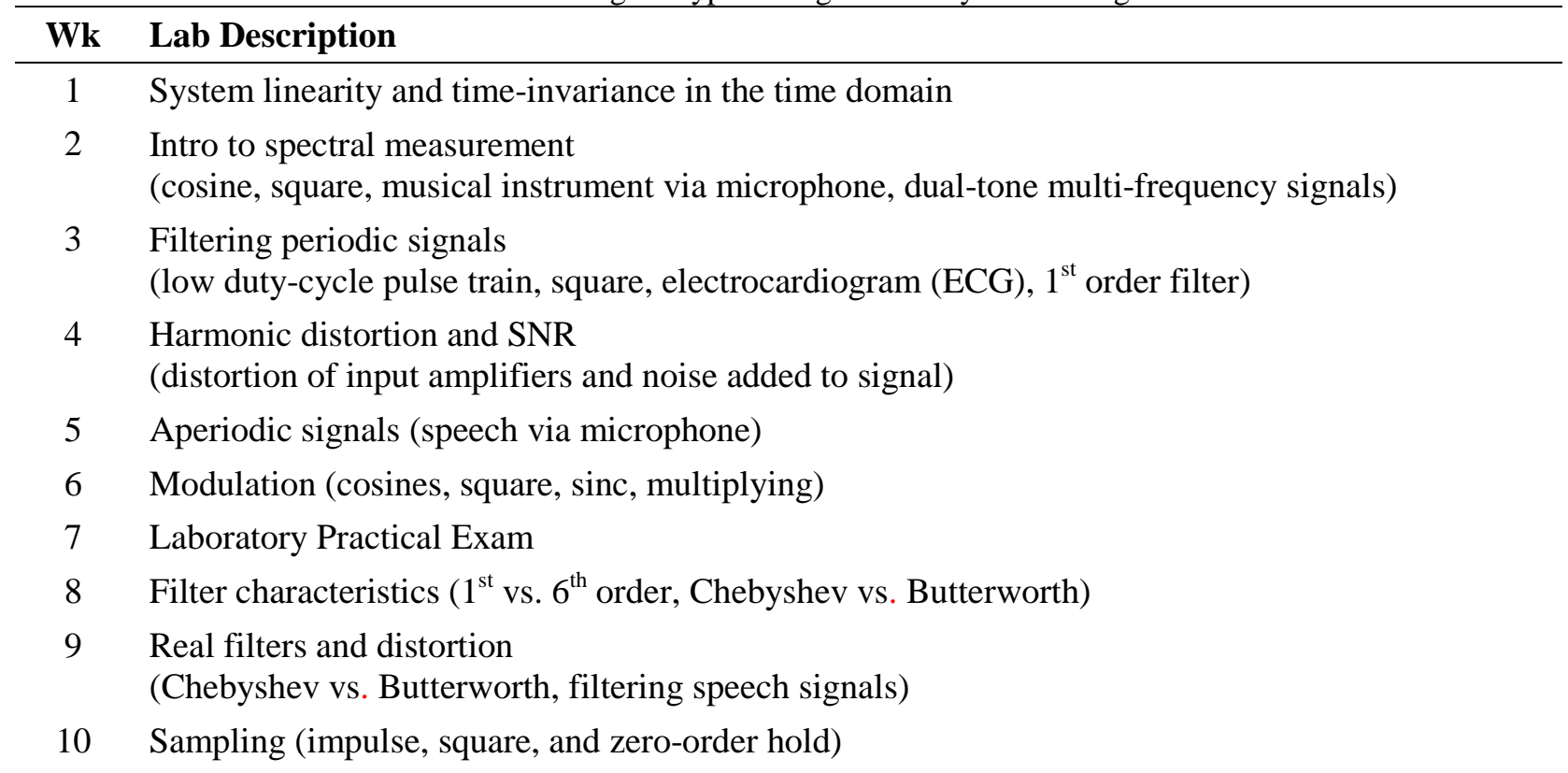

\section{Lab 1: System Properties in the time-domain}

The first lab provides students with an experience of time domain system properties such as linearity, time invariance, and impulse response. The point of the lab is to introduce students to the SSEP and provide them with an experience of system properties. Linearity and impulse response are issues that are relevant through the entire quarter. The students need to be aware that their data is invalid if the amplifiers saturate, and what limitations are necessary to prevent the system from saturating. Students begin the lab with simple signals such as triangle waves that allow them to measure these limitations under different conditions such as homogeneity and then when two different signals are summed together. Then the students use the on-board multiplier and vary the multiplying signal to understand the difference between a time-varying and timeinvariant system. For both of these exercises, the students can play the distorted signals on a speaker so that they can hear the change in frequency content. Finally, students are asked to measure the impulse and step response of the system by using very low duty-cycle periodic pulse signals to approximate impulses and 50\% duty-cycle square waves to approximate a step signal. By comparing the step and impulse responses to what is expected of first and second order systems, the students can get an appreciation for the order of the system. It is indicated that the order of the system has influence on the filtering properties of the system. At the end of the lab, students are asked to describe, based on their measurements, the limitations that are necessary to ensure that the system will behave like a linear-time-invariant system in future labs. 
While these exercises are designed to be completed in a three hour session, not all schools have laboratory components to their introductory CTSS courses. The exercises can be broken up into smaller steps and those steps done as demonstrations in a class setting. For example, time invariance can be shown by using the SSEP to multiply an approximated impulse train with different signals. The first signal could be a DC voltage to show a time-invariant system by illustrating that the impulse response doesn't change from one impulse to the next. Then a timevarying signal can be used to show that the impulse response changes depending on when the impulse is applied. Changing the gain on the SSEP by turning a potentiometer can then illustrate the difference between a linear and nonlinear system by causing the amplifiers to go into saturation for some of the impulses. This whole exercise can be done with the SSEP in just a few minutes by the instructor and in less than 30 minutes by the students.

One advantage of using the SSEP is that it is a very flexible and general platform. Another idea is to create a delay line of 3 first-order systems and model it using mathematics. Students could play both the original and delayed signal through a speaker to model an echo. Different loads, such as electric motors and solenoids, could be connected to the output of the SSEP to see how they affect the linearity and time invariance of the system. The ideas presented in this paper are just a first attempt, and are meant to spark discussion with other instructors to create even more meaningful activities for the students.

\section{Lab 2: Introduction to Frequency Domain Measurements}

The second lab introduces students to spectral measurements using periodic signals. They start with a simple sinusoid and observe how the three characteristics of amplitude, frequency, and phase relate to such measurements. Then the SSEP is used to sum two sinusoids together and the results are observed in both the time and frequency. When the sinusoids have the same frequency, it is observed that the phase difference can impact power measurements, but when the frequencies are different, the phase does not impact the power. Once the students can demonstrate the ability to measure and understand two sinusoids, more complicated signals such as square and triangle waves can be used. Two real-world applications are used to demonstrate the usefulness of frequency domain measurements. The first is focused on musical notes recorded with the microphone that is on the SSEP. The students blow into a plastic bottle and then two different lengths of a straw flute to make notes that can then be observed on the oscilloscope and spectrum analyzer. The second application is to observe the output of a DTMF signal generator ${ }^{4}$. These are readily available from older touch-tone phones or even online DTMF generators that could be played from the sound card of a laptop. For the standardized waveforms, the students are asked to compare their measurements to predicted values that they calculate using the Fourier series coefficients. For the musical notes, they are asked to describe what makes a D4 note played on a piano and guitar different even though they have the same fundamental frequency.

\section{Lab 3: Filtering Periodic Signals}

The third lab introduces students to analyzing systems in the frequency domain. A simple firstorder op-amp based RC filter is used with periodic signals. The students look at very short duty cycle pulse trains to approximate a periodic impulse train. Within the bandwidth they are using, they observe that the components at each harmonic have approximately the same power. When 
they put the signal through a first order filter, they notice two things. First, the powers at each harmonic change due to the frequency-dependent gain of the filter. Second they can see the impulse response of the filter. The students can then change the fundamental frequency of the pulse train and observe how the gain at each harmonic changes. The next step is to filter a slightly more complex signal, which is a 50\% duty cycle square wave again at different fundamental frequencies. For an application, the students measure their own ECG signal, which is typically very noisy. When they pass the signal through the first order filter, they can suddenly see the signal and realize what the filter does. One of the questions that we ask the students is why the filter removes the noise but lets the ECG signal through unaltered.

\section{Lab 4: Harmonic Distortion and SNR}

The fourth lab introduces students to more complicated frequency domain measurements of signals and systems, harmonic distortion (HD) and signal-to-noise ratio (SNR). Because the students are using analog electronics, these electronics will naturally distort the signal as the amplitude increases. The students start off with a small amplitude sinusoid and measure the power in the harmonics as they increase the amplitude. When they plot the HD with respect to input amplitude, they can get an idea of how to quantify nonlinearity from a frequency domain perspective. While taking these measurements, the students can listen to the signal on a speaker and hear the harmonics being added to the signal as it begins to get distorted. Many students also see concepts such as large and small-signal modeling in their electronics courses and this helps to tie those concepts together. After doing the experiment students can be asked questions such as why it is difficult if not impossible to measure harmonic distortion with a triangle wave instead of a simple sinusoid. To measure signal to noise ratio, the students first pass a noise signal from a function generator through a $1^{\text {st }}$ order filter that provides a known signal bandwidth. They measure the power in the time-domain using a DMM to provide the RMS voltage and then squaring the value. As they increase the noise amplitude on the function generator, they can see how the noise power changes in the time and frequency domains. Then they add to the noise a fixed amplitude sinusoidal signal as they go through the same changes in noise amplitude and observe what the combined signals look like at different signal-to-noise ratios. Furthermore, they can listen to the sounds on a speaker, which is a more familiar experience with regard to this concept because everyone has experienced static on a cell phone. The SNR measurements are repeated for a filter with a lower cutoff frequency and then again for a fixed amplitude ramp signal. They can observe how the noise floor in the spectrum rises with an increase in noise power and can overcome certain harmonics in the signal, which are no longer heard. The students are then asked to reflect on what happens when the signal-to-noise ratio has a very large positive or negative $\mathrm{dB}$ value.

\section{Lab 5: Frequency Content of Aperiodic Signals}

The fifth lab introduces the Fourier transform for aperiodic signals. By this time the students are more comfortable with frequency domain measurements and have a better understanding of how a signal is composed of cosines with different frequencies. This lab starts by illustrating the relationship between the Fourier series and transform. Students start with a periodic square wave with 50\% duty cycle and then gradually decrease the fundamental frequency of the signal without changing its pulse width. The oscilloscope is then set up to display both the time-domain version of the signal and its FFT. As the signal is altered, the students can see the increase in the 
number of harmonics and the change in spacing between the harmonics as the frequency decreases. Because the oscilloscope takes the FFT only of the portion of the signal that is on the screen, when only a single pulse is shown, the spectrum merges into a single continuous function that has the same shape as the Fourier transform, although a different vertical scale. Then the students start to work with speech signals that are recorded via the microphone. The oscilloscope is used to get a high-resolution sample of the speech signals and then the data is downloaded onto the computer. The experiments focus on the word "these”. Each syllable, "th”, "ee”, and "sss", is captured individually and then the whole word while prolonging each syllable, "ttttthhhhheeeeeeesssss". Using the "sss" sound instead of "zzz" for the final syllable enables the students to see the difference between noise and well-defined frequencies. The oscilloscopes that are used permit the downloading of the actual data points into a CSV file that can then be loaded into MATLAB ${ }^{\circledR}$. The students then plot the FFT of each signal in order to observe its magnitude and phase spectrum, which is not possible on the oscilloscope. They also plot the spectrogram of each syllable and then the whole word. Using the spectrogram and FFT of each of the signals, the students are asked to describe the difference between looking at the frequency spectrum of a signal as a whole versus breaking it up into small time fragments and observing how the frequency content changes over time.

\section{Lab 6: Modulation}

The sixth lab focuses on modulation as an example of the Fourier transform. The students use the multiplier on the SSEP to modulate several different signals. They start with the multiplication of two sinusoids to see how modulation creates the sum and difference frequencies. By varying the frequency of one of the sinusoids and observing the effects in real-time, they can get a sense of the frequency shifting nature of modulation. Then the students modulate a rectangle and sinc function and see how the copies of the spectra in the frequency domain can interact if the modulation frequency is too small or if the signal is not bandlimited. Finally, they modulate a version of the word "these" that is stored on the function generator. The word only sounds natural at a specific fundamental frequency on the function generator. By varying this frequency, the students can experience the difference between frequency scaling versus modulation as a method for shifting the frequencies of the signal. When played on a speaker, the students also hear the difference between these two methods. They can experience how modulation distorts the harmonic relationship between the frequencies and how this effect worsens as the modulation frequency increases. The students are asked to describe why a DC offset added to a modulated signal does not show up at $\mathrm{OHz}$ on the spectrum.

\section{Lab 7: The Lab Practical}

During the seventh week of the quarter the students are given a practical exam that evaluates the students' ability to use the equipment and analyze signals. This exam is composed of three components: time domain measurements, frequency domain measurements, and the ability to program in MATLAB ${ }^{\circledR}$. Students are asked to analyze screen captures of the oscilloscope and spectrum analyzer and then make frequency domain measurements. For the MATLAB ${ }^{\circledR}$, the students are asked to write a simple script from a blank page and then plot the results. The script usually involves the calculation and plotting of a Fourier series given the coefficients. 


\section{Lab 8: Characterizing Filters}

The last three laboratory sessions are based on applications of the frequency domain involving filtering and sampling. In the eighth lab, the students characterize four different analog filters by measuring first their impulse response and then the change in amplitude and phase of a single sinusoid at different frequencies. All filters are lowpass and made with resistors, capacitors, and operational amplifiers. The amplifiers are pre-wired on the SSEP for $2^{\text {nd }}$ order multiple-feedback filter stages so that the students only have to insert the passive elements into fixed connectors. The four different types are $1^{\text {st }}$ order, $6^{\text {th }}$ order Butterworth, $6^{\text {th }}$ order Chebychev with $2 \mathrm{~dB}$ of ripple in the passband, and $6^{\text {th }}$ order Bessel. As a prelab exercise, the students are asked to plot the impulse response, magnitude of the frequency response on a linear and $\mathrm{dB}$ scale, phase of the frequency response, and delay of the frequency response. The impulse response is measured by using a very small duty-cycle pulse and the response is compared to what is provided by MATLAB $^{\circledR}$ for that filter. Then a single sinusoid is input to each filter and the students measure the change in amplitude and phase at various frequencies. The results are then compared to the ideal plots provided by MATLAB ${ }^{\circledR}$. To do all of these activities takes the full three hour session, so that using the filters in an application occurs in the following week.

\section{Lab 9: Using Continuous-Time Filters}

In the ninth lab, the students use the filters that were characterized in the previous week. They first input a signal that consists of four equal-amplitude cosines that are in phase and harmonically related (i.e $\left.x(t)=A \cos \left(\omega_{0} t\right)+A \cos \left(2 \omega_{0} t\right)+A \cos \left(3 \omega_{0} t\right)+A \cos \left(4 \omega_{0} t\right)\right)$. This signal is stored on the function generator so that the students can adjust its amplitude and fundamental frequency. Because the filters have a fixed -3dB frequency, as the fundamental frequency of the signal is increased, some harmonics will be located in the stop-band of the filters. The students then observe how the frequencies in the spectrum change and the relationship of this change to the signal in the time domain. After using this simple signal, the students are asked to filter the word "these" as played back from the function generator. By comparing back to the spectrogram of the word, the students can see which frequencies of the signal are being filtered out. Each of the filters has different effects on the signal and the students are asked to explain these differences.

\section{Lab 10: Sampling}

The tenth lab focuses on sampling and uses the multiplier and sample-and-hold circuits on the SSEP to perform three different forms of sampling: ideal impulse sampling, multiplying by a $50 \%$ duty-cycle square wave, and zero-order-hold $(\mathrm{ZOH})$ sampling. For each of these cases, the students are asked to sample the signal from the previous lab that is made of four harmonicallyrelated cosines. The fundamental frequency of the input signal is fixed and then the students vary the sampling frequency and the type of filter used to recover the sampled signal. At the higher sampling frequencies, there is no aliasing of the copies of the spectrum of $\mathrm{x}(\mathrm{t})$ in the frequency domain, but at the lowest sampling frequency the copies do alias. They use the $1^{\text {st }}$ order and $6^{\text {th }}$ order Butterworth filters to try to recover the original signal. For each case, the students observe the sampled signal in the time and frequency domains simultaneously so that they can see what aliasing looks like in each domain. They are able to see the difference between aliasing that is due to a violation of the Nyquist criterion versus aliasing that results from the filter not having 
sufficient attenuation. The students are asked to identify several features in the frequency domain measurements such as the copies of the spectrum of $x(t)$, the harmonics of the sampling frequency, and any indications of aliasing. They are also asked to describe the differences between the three forms of sampling and why one might be better than the other.

\section{Assessment}

The primary purpose for creating these laboratory exercises was to give students a personal experience with the theoretical concepts and to show them how the theory can be applied in meaningful ways. If the students could gain these experiences, then we hypothesized that their attitudes, and consequently learning, in the course will improve. Two assessment tools were used to measure the impact of the laboratory exercises on the students. In order to assess the students' affective learning experience a survey was administered for one year before the exercises were introduced and also for every quarter in which the exercises were used during the past few years. The Continuous-Time Signals and Systems Concept Inventory (CTSSCI) is used to assess any changes in the students' cognitive learning experience. The exam is a measure of the students' understanding of the basic concepts in the course. One of the purposes of the lab exercises is to help the students gain a more intuitive understanding of the concepts. In this sense the CTSSCI is a good match to this study.

\section{TABLE II}

Results from three questions that were taken from a survey that was administered for one year before the new lab exercises were introduced, as indicated by the "Mean Prior" column, and then for all quarters in which they have been used, as indicated by the "Mean During" column. A two-tailed, type-2 t-test was used to determine the statistical significance of the change in means from prior to during.

\begin{tabular}{|c|c|c|c|c|}
\hline & $\begin{array}{l}\text { Questions with corresponding range of choices and } \\
\text { associated values for each end of the range }\end{array}$ & $\begin{array}{l}\text { Mean } \\
\text { Prior } \\
(n=36)\end{array}$ & $\begin{array}{l}\text { Mean } \\
\text { during } \\
(\mathrm{n}=68)\end{array}$ & p-value \\
\hline 1 & $\begin{array}{l}\text { I think the concepts that I learned in ECE300 were } \\
\text { (1) All theoretical - } \\
\text { (5) All application oriented }\end{array}$ & 2.2 & 2.65 & 0.0005 \\
\hline 2 & $\begin{array}{l}\text { The labs in ECE300 helped to motivate the material and explain why it is important. } \\
\begin{array}{ll}\text { (1) strongly agree - } & \text { (5) strongly disagree }\end{array}\end{array}$ & 2.58 & 2.10 & 0.02 \\
\hline 3 & $\begin{array}{l}\text { Please rate your confidence with using Fourier Series or Fourier Transforms and a system's } \\
\text { impulse response to determine the system's output in the time and frequency domain } \\
\text { (1) very confident - (4) very unconfident }\end{array}$ & 1.72 & 2.01 & 0.04 \\
\hline
\end{tabular}

The survey contained 13 questions that were intended to measure perceptions about the concepts, perceptions about the exercises themselves, and confidence with the material. Of the original 13 questions, three demonstrated statistically significant changes in the mean from before and after the exercises were introduced. It is serendipitous that each question represents one of the three different themes in the survey. The results from these three questions are given in Table II. The purpose of the first question was to measure any changes in the students' perception of the material. While CTSS courses are math intensive, the purpose of the math is to help students understand and solve real-world problems from a frequency domain perspective. The results from these questions could indicate if the exercises are helping the students to see how the 
concepts can be useful to engineers. The results of question 1 show a significant change $(\mathrm{p}<0.01)$ in the mean by approximately $10 \%$ towards the students seeing more application of the material.

The second question on the survey was meant to determine if the new analog-circuit based exercises were having an impact on that shift in perspective. Prior to implementing the new exercises, the lab sessions were based primarily on MATLAB ${ }^{\circledR}$ simulations. Question 2 shows a significant change $(\mathrm{p}<0.05)$ of approximately $10 \%$ towards the students agreeing that the lab exercises help to motivate the material. This result indicates that the new labs are helping the students to understand why the material is important to them.

The third question is intended to measure changes in student confidence with the material as a result of performing the new exercises. This question shows a significant change $(p<0.05)$ of approximately $10 \%$ in the direction of being less confident with the material. While technically in the wrong direction, a value of 2 for this response still indicated "somewhat confident" with the material. It's possible that some key element was lost when switching to the new labs or that the new exercises were more complicated, so that students did not feel as confident when performing them. In any case, it warrants further study to understand why students would feel less confident in the material.

Understanding the Concept Inventory results requires some historical perspective on how ECE300 has changed over the past few years. These changes are described in the timeline in Figure 2. Prior to the Winter Quarter of the 2009-2010 academic year, both the time- and frequency-domain material was part of ECE300. This original version of ECE300 had existed for approximately 6 years prior to that term. In the Winter Quarter of 2009-2010, the time-domain material was removed from ECE300 and put into a pre-requisite course. Then the new labs were introduced in the Fall Quarter of the 2010-2011 academic year. The result is that there were two quarters in which the new version of ECE300 was taught using the old MATLAB ${ }^{\circledR}$-based labs.

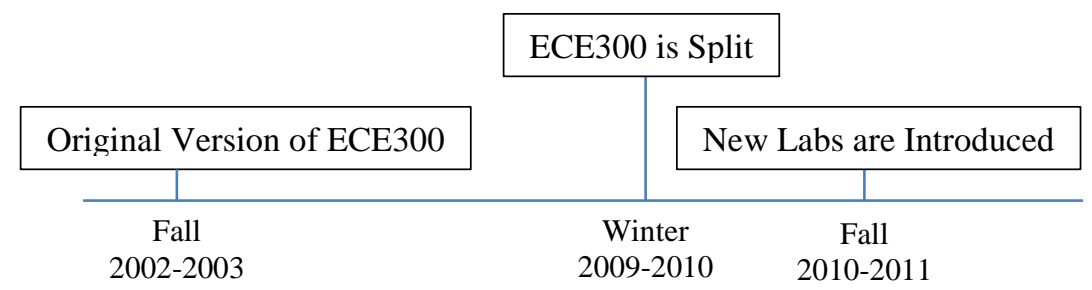

Figure 2: Timeline of changes that were made to ECE300

The CTSSCI consists of 25 questions related to time- and frequency-domain and Laplace analysis of signals and systems ${ }^{6}$. The breadth of questions contained on the concept inventory was never completely covered in ECE300, even under the original version of the course. When the course was changed in the Winter Quarter of 2009-2010, the subset of questions that were covered in ECE300 also changed. In order to understand the impact of ECE300 on the concept inventory results, a subset performance is reported in addition to the performance on the whole exam. The subset consists of those specific questions that were covered in the version of ECE300 for which the concept inventory was given. For the original version of ECE300, this subset consists of 20 questions, while for the new version only 16 questions were used. 
The results of the concept inventory are shown in Table III. The score reported represents the average normalized gain of the students in that group. The p-value is the result of a two-tailed ttest to evaluate the change in mean from the group of all students prior to the winter of AY20092010 (i.e. the first row). The normalized gain is defined as (post-pre)/(\#ques-pre), where post and pre are the individual student's score on the pretest and posttest and \#ques is the number of questions being evaluated. The normalized gain of each student was computed first and then the average taken. For the subset score the \#ques term in the normalized gain was adjusted to either 16 or 20 as described previously. For the All Questions score the \#ques term was 25, which accounted for all of the questions on the exam. The Avg Class Grade/4.0 column represents the average grade in ECE300 for all students in that group out of a 4.0 scale with $4.0=\mathrm{A}, 3.5=\mathrm{B}+$, 3.0 $=\mathrm{B}, 2.5=\mathrm{C}+, 2.0=\mathrm{C}, 1.5=\mathrm{D}+, 1.0=\mathrm{D}, 0.0=\mathrm{F}$, and $0.0=$ Withdraw.

The benchmark to which all changes are compared is the original version of ECE300 (first row of Table III), for which 8 years' worth of data is reported. Even though the subset of questions changed between groups, this group was used as the basis of comparison in order to have a larger data set. Furthermore, because the normalized gain is being used as the measure of performance, the impact of the different subsets is somewhat lessened.

The second row of Table III indicates the impact of changing the curriculum in the course without the new labs. The means of normalized gain for both the subset and total score did not change considerably from the basis group. It is difficult to determine the impact that the curriculum change had on student performance based on this data alone. The mean of each normalized gain did not change much, the result is not statistically significant ( $\mathrm{p}>0.1$ ), and the group size is small. Furthermore, the first time a course is offered rarely provides the best assessment data because of all the transients that arise during course development.

TABLE III

Results from the Continuous Time Signals and Systems Concept Inventory for all students taking ECE300 during the past 10 years.

\begin{tabular}{|l|c|c|c|}
\cline { 2 - 4 } \multicolumn{1}{c|}{} & $\begin{array}{c}\text { ECE300 } \\
\text { Subset }\end{array}$ & $\begin{array}{c}\text { All } \\
\text { Questions }\end{array}$ & $\begin{array}{c}\text { Avg Class } \\
\text { Grade/4.0 }\end{array}$ \\
\hline $\begin{array}{l}\text { Original Version of ECE300 (n=680) } \\
\text { Through Fall of 2009-2010 }\end{array}$ & 0.42 & 0.31 & 2.5 \\
\hline $\begin{array}{l}\text { Year Prior to labs (n=25) } \\
\text { Winter and Spring 2009-2010 }\end{array}$ & $\begin{array}{c}0.38 \\
(\mathrm{p}=0.53)\end{array}$ & $\begin{array}{c}0.28 \\
(\mathrm{p}=0.59)\end{array}$ & 2.75 \\
\hline $\begin{array}{l}1^{\text {st }} \text { year of labs (n=75) } \\
\text { All three quarters of 2010-2011 }\end{array}$ & $\begin{array}{c}0.30 \\
(\mathrm{p}=0.00)\end{array}$ & $\begin{array}{c}0.18 \\
(\mathrm{p}=0.00)\end{array}$ & 2.45 \\
\hline $\begin{array}{l}2^{\text {nd }} \text { year of labs }(\mathrm{n}=81) \\
\text { All three quarters of 2011-2012 }\end{array}$ & $\begin{array}{c}0.54 \\
(\mathrm{p}=0.00)\end{array}$ & $\begin{array}{c}0.36 \\
(\mathrm{p}=0.13)\end{array}$ & 2.35 \\
\hline
\end{tabular}

The last two rows illustrate the change in performance on the concept inventory after the new lab exercises were introduced. They are separated by year because of the significant change from one year to the next. Note that in the first year in which the new labs were used the performance on the concept inventory actually went down relative to the basis group. However, in the second 
year, the performance increased substantially especially with regard to the subset questions. The same faculty member taught the course throughout both years with approximately the same curriculum. The average class grade is given to indicate that the students in the 2011-2012 academic year were not necessarily more gifted.

There was one major change made to the laboratory exercises between the first and second year that could have contributed to this difference. During the first year of using the new exercises, they were still being developed and were a bit awkward to complete. Some of the consistent feedback from the students indicated that they didn't see the purpose of running through all of the detailed instructions. They were getting bogged down in performing the exercises and not seeing how it related to the material. The short answer questions being asked at the end of the lab were too difficult to answer and too removed from the experiment.

In the summer between the academic years, two major changes were made to the laboratory materials. First, the instructions were simplified and made easier to follow. Second, thought provoking questions were added to all of the major steps of each exercise rather than being placed only at the end of the lab. Students were informed that these intermediate questions were the main purpose of doing the exercises, and that it was expected that they be able to answer them should the instructor visit their station. By observation of the instructor during the second year, many more students were pausing during the lab and thinking about what was happening during the exercises rather than simply trying to race through the instructions, collect the data, and leave. This begs the question of whether or not it is even necessary for students to collect data, or would it be more beneficial to simply answer a series of short answer questions?

\section{Summary}

A new set of laboratory exercises were developed to improve learning in an introductory continuous-time signals and systems course. These exercises provide an opportunity for students to associate the highly mathematical and theoretical modeling concepts with real-world problems through hands-on activities. Because the experiments are conducted with analog circuits, the students can manipulate the signals and systems in real-time while simultaneously observing the results in both the time and frequency domains. Such associations can help the students to relate to, visualize, and understand the concepts. While these exercises were designed for and facilitated by a specialized analog circuit platform, it is possible to do them with standard analog circuits and measurement equipment or even computer mediated equipment such that offered by National Instruments. The topics covered by the labs include system properties, frequency domain measurements, modulation, filters, and sampling.

The underlying hypothesis for creating these exercises is that if students can better relate to and visual the concepts, then learning and attitudes towards the material will improve. The labs definitely helped the students to gain a more application-oriented perspective of the concepts, but their confidence with the material did not change much. The concept inventory results suggest that, explicitly mandated reflection during the experiment can have an impact on student understanding. We will continue to improve the labs by adding design options and more time for reflection, while measuring the impact of these changes. More detailed studies are already under development to determine best practices for these experiments. One factor that would be interesting to study is the impact of the hands-on nature of the experiments relative to a 
simulation only based experiment. The advantage of doing simulations only is the reduced cost and accessibility to the students, but what is lost is the connection to a physical system. It will be interesting to see what impact the hands-on aspect has with regards to CTSS concepts in particular.

\section{Acknowledgements}

This work is funded by NSF grant \#1140995. Special thanks to Shannon Sexton in the IRPA office at Rose-Hulman for her help with the assessment section.

\section{References}

[1] J. Nelson, M. Hjalmarson, K. Wage, and J. Buck, "Students' interpretation of the importance and difficulty of concepts in signals and systems," in Frontiers in Education Conference (FIE), 2010 IEEE, Oct. 2010, pp. T3G-1 -T3G-6.

[2] K. Wage, J. Buck, and C. Wright, “Obstacles in signals and systems conceptual learning,” in Digital Signal Processing Workshop, 2004 and the 3rd IEEE Signal Processing Education Workshop. 2004 IEEE 11th, Aug. 2004, pp. $58-62$.

[3] M. Simoni, "Work in progress - A hardware platform for a continuous-time signals and systems course", Proceedings IEEE Frontiers in Education Conference (FIE), 2011, pp. S4G-1 - S4G-2.

[4] M.F.Aburdene and K. Nepal, "Wow! linear systems and signal processing is fun!”, Proceedings IEEE Frontiers in Education Conference (FIE), 2011, pp. F1G-1 - F1G-6.

[5] L. G. Huettel, "A dsp hardware-based laboratory for signals and systems,” in IEEE 12th Digital Signal Processing Workshop and 4th Signal Processing Education Workshop, Moose, WY, United States, 2006, pp. 456 459.

[6] J. Ma and J.V. Nickerson, "Hands-On, Simulated, and Remote Laboratories: A Comparative Literature Review”, ACM Computing Surveys, Vol. 38, No. 3, Article 7, September 2006.

[7] J.E. Corter, J.V. Nickerson, S.K. Esche, C. Chassapis, S. Im, and J. Ma, "Constructing Reality: A Study of Remote, Hands-On, and Simulated Laboratories”, ACM Transactions on Computer-Human Interaction, Vol. 14, No. 2, Article 7, August 2007.

[8] K. E. Wage, J. R. Buck, T. B. Welch, and C. H. Wright, "The continuous-time signals and systems concept inventory," in Proceedings of ICASSP, IEEE International Conference on Statistical, Speech, and Acoustic Signal Processing, vol. 4, Orlando, FL, United States, 2002, pp. IV/4112 - IV/4115. 